\title{
Breast Pathologies and Inadequate Breastfeeding Practices: A Survey Among a Group of Newly Delivered Women in Yaoundé, Cameroon
}

\author{
Georges Pius Kamsu Moyo \\ Faculty of Medicine and Biomedical Sciences, University of Yaounde 1, Yaounde, Cameroon
}

\section{Email address:}

kamsuzicfried@yahoo.fr

\section{To cite this article:}

Georges Pius Kamsu Moyo. Breast Pathologies and Inadequate Breastfeeding Practices: A Survey Among a Group of Newly Delivered Women in Yaoundé, Cameroon. Journal of Family Medicine and Health Care. Vol. 6, No. 3, 2020, pp. 87-90.

doi: $10.11648 /$ j.jfmhc.20200603.16

Received: June 22, 2020; Accepted: July 7, 2020; Published: July 23, 2020

\begin{abstract}
Breastfeeding women may develop a number of breast disorders during the postpartum period. Such disorders could involve masses of varying sizes, breastmilk secretion anomalies and painful-inflammatory or infectious conditions. Though very few of these conditions may be absolute contraindications to breastfeeding, they are however a significant source of worry for newly delivered women and may disrupt the breastfeeding process. This survey aimed to investigate breast anomalies in women with inadequate breastfeeding practices. We conducted a cross-sectional study from December 2018 to May 2019 at the Yaoundé Gynaeco-Obtetric and Paediatric Hospital. Mothers with livebirth neonate infants weighing $>2000 \mathrm{~g}$ and with no initial contraindication to breastfeeding were included. A total of 250 mothers were enrolled in the survey. Delivery was eutocic in 230 (92\%) women and 199 (79.6\%) had the intension to exclusively breastfeed their babies. The state of the neonates was satisfactory in $204(96 \%)$ deliveries. However, inadequate breastfeeding was noted in 153 mothers $(61.2 \%)$, with breast pathologies occurring in 85 women (55.5\%), among which $24(28.2 \%)$ had secretion anomalies predominated by quantitative disorders. Painful inflammatory and/or infectious conditions occurred in 58 women (69.4\%) including nipple crevices, breast engorgement, inverted nipples, mastitis and abscess. All women with obvious or supposed breast disorders had inadequate breastfeeding practices. Therefore, painful breast pathologies and secretion disorders should firstly be investigated in women with eutocic delivery, who are willing to breastfeed, but unable to do so adequately. More so, these pathologies are essentially benign and may be prevented by good hygiene, adequate breastfeeding techniques such as proper nipple and areolar positioning into the baby's mouth.
\end{abstract}

Keywords: Breast Pathologies, Breastfeeding, Lactation

\section{Introduction}

Inadequate breastfeeding may be defined as a process characterized by either a delayed initiation, unexclusiveness, ineffectiveness, briefness or short lasting breastfeeding. The timely initiation of breastfeeding, also known as early initiation of breastfeeding (EIBF) refers to the proportion of newborn infants who are breastfed within the first hour following delivery [1]. The World Health Organization recommends that breastfeeding should be initiated within 30 minutes to an hour at most after childbirth $[1,2]$. In effect, breastfeeding in mothers is a physiological process which may be broken down into two components. On one side, we have the physical act that depends on endocrinal stimulation from prolactin which is primordial for breastmilk secretion, and oxytocin for milk ejection. On the other hand, is the mental or psychological component which relies on both affective or emotional interactions with possible endocrinal enhancement due to endorphin. In infants, suckling the mother's nipple provokes breastmilk ejection, which is then digested and serves for immunological protection, optimal physical, neurological and psycho-affective development. The WHO thus recommends exclusive breastfeeding during the first six months of life as being fundamental for an optimal growth [2, 4-6]. Even though there is considerable effort put in place especially in developing countries to 
improve breastfeeding practices, there are however some particular conditions, including breast disorders that may hinder the process [3]. Indeed, during the postpartum period, the breast may be affected by a number of specific disorders including physiological and/or anatomical impairments. Breast pathologies may either be benign, malignant, inflammatory, infectious or a combination of various process [7]. Nevertheless, some common breast pathologies that develop in lactating women include inverted nipples, nipple cracks, fissures and crevices, breast engorgement, mastitis, and abscess. Painful inflammatory or infectious conditions, represent up to $70 \%$ of breast pathologies during this period [7]. Whereas, benign focal masses involve fibroadenomas, lactating adenomas, galactoceles and papillomas, while breast adenocarcinoma is the most frequent malignant tumor [7]. This survey aimed at enquiring the most frequent breast disorders in women with inadequate breastfeeding practices in our context.

\section{Methodology}

We conducted a cross-sectional study with prospective data collection, over a six-month period from December 2018 to May 2019, at the Yaoundé Gynaeco-Obstetric and Paediatric Hospital which is a University Teaching Hospital in Cameroon. We included all newly delivered women with livebirth newborn infants weighing more than $2000 \mathrm{~g}$, with no initial contraindication to breastfeeding and who consented to participate in our study. The enrolled mothers were observed during the first 2 weeks of postpartum to detect those that would practice adequate breastfeeding in terms of the timely initiation, the effectiveness of breastfeeding technique, exclusiveness and duration in conformity with the WHO's recommendations. Breast disorders were eventually sought to identify the most occurring ones. The data were analyzed using CS Pro version 6.2 and SPSS version 20.0.

Ethical clearances from the Institutional Ethics and Research Committee of the Faculty of Medicine and Biomedical sciences of the University of Yaoundé 1, and the Yaoundé Gynaeco-Obstetric and Paediatric Hospital were obtained before the beginning of the survey. The data collected was kept confidential and used for the purpose of the study only.

\section{Results}

A total of 250 mothers were enrolled in the survey, the mean age was $27.9 \pm 6.2$ years. Close to 174 women $(69.6 \%)$ had at least 02 living children, 208 women (83.2\%) had a school education level $\geq$ secondary education level.

Delivery was eutocic in 230 (92\%) women and 199 (79.6\%) had the intension to exclusively breastfeed their babies. The state of the neonates was satisfactory in 204 (96\%) deliveries. However, inadequate breastfeeding was noted in 153 mothers $(61.2 \%)$. Breast pathologies occurred in 85 of such women (55.5\%), among which 24 (28.2\%) had secretion anomalies predominated by quantitative disorders. Painful inflammatory and/or infectious conditions occurred in 58 women (69.4\%) including nipple crevices, breast engorgement, inverted nipples, mastitis and abscess. All women with obvious or supposed breast disorders had inadequate breastfeeding practices

The various breast pathologies found in women with inadequate breastfeeding practice are summarized in table 1 below.

Table 1. Breast pathologies in women with inadequate breastfeeding.

\begin{tabular}{lll}
\hline Breast disorder & N (85) & Percentage (\%) \\
\hline Secretion anomalies & 24 & 28.2 \\
Nipple crevices and fissures & 21 & 24.7 \\
Breast engorgement & 15 & 17.6 \\
Inverted nipples & 09 & 10.6 \\
Mastitis & 07 & 8.2 \\
Abscess & 07 & 8.2 \\
Lactating adenoma & 02 & 2.3 \\
\hline
\end{tabular}

Table 2. Breastmilk secretion disorders.

\begin{tabular}{lll}
\hline Breast secretion disorder & N (24) & Percentage (\%) \\
\hline Quantitative secretion disorder: & 15 & 62.5 \\
1st day agalactorrhea & 08 & 33.3 \\
Hypogalactorrhea & 07 & 29.1 \\
Qualitative secretion disorders: & 09 & 37.5 \\
Pus stained breastmilk & 07 & 29.1 \\
Blood stained breastmilk & 02 & 8.3 \\
\hline
\end{tabular}

\section{Discussion}

From this survey, it appeared that age maturity and school education alone may not be sufficient enough to guarantee adequate breastfeeding in mothers. This could be deduced from the fact that, even though more than $50 \%$ of women enrolled had an education level greater than secondary school, and close to $69 \%$ had past experience of breastfeeding, yet the level of inadequate breastfeeding was as high as $61.2 \%$ which is quite much, as compared with other African developing countries [8,9]. All the same, this rate was neither improved by the fact that up to $92 \%$ of deliveries were without complications and that in more than $79.6 \%$ of cases, the mother-infant couple had good predispositions for breastfeeding. This permitted to suspect that the breast and its related issues were a main obstacle to breastfeeding in our series. However, among 55.5\% mothers with inadequate breastfeeding practices who had breast disorders as well, breastmilk secretion anomalies which were either quantitative or qualitative, were the most predominant in $28.2 \%$ women, when all factors were assessed separately. Whereas, on a cumulative basis, painful conditions including nipple crevices, breast engorgement, inverted nipples, mastitis and abscess, in order of frequency, occurred in over $69 \%$ women with breast disorders. Breast tumors were rare in our series, with the benign lactating adenoma diagnosed in $2.3 \%$ women.

Quantitative secretion disorders involved initial agalactorrhea and hypogalactorrhea which are most frequent during the immediate postpartum period [10]. Amelioration of breastmilk synthesis was noted thereafter, with continuous nipple stimulation by baby suckling and a balanced diet for the mother, 
including supplementary hydration. Qualitative anomalies involved nipple discharges which were either isolated or mixed with breastmilk, producing particular staining. Yellow and green discharges were associated with abscess and were unilateral, while rusty brown and bloody discharges were bilateral, intermittent and spontaneously resolving [11, 12]. Bloody discharges in this survey were physiological, due to hormoneinduced hyper vascularization, congestion, structural and functional epithelial modifications [7, 13]. This differs from unilateral bloody discharges which are generally associated with nipple trauma including crevices [14].

Breast engorgement as defined in this study was considered as overfilled breasts becoming hard, edematous, and painful, with transient fever, nipple flattening or inversion, and the overall modifications altering the breastfeeding process [15]. In effect, there existed a vicious cycle between engorgement and other breast pathologies as described in the literature. Women with breast engorgement who were unable to breastfeed, later on developed aggravation, nipple crevices, inversion and mastitis with further inflammation $[15,16]$. Such women were thought breast emptying techniques including shower before expressing, warm compress application over the breast few minutes before pressing [16, 17]. Whereas, women with engorgement, and able to breastfeed were encouraged to do so more frequently in order to prevent accumulation [16, 17].

There were two forms of acquired inverted nipples: retracted nipples were only partly inverted, whereas invaginated nipples were completely inverted. Nipple inversion in lactating women is most often secondary to underlying inflammatory changes [18]. Short-lasting acute inversion were more frequent in this survey. Just as in many similar research studies, women with inverted nipples were more likely to suffer from mastitis and infectious complications [18]. However, Nipple cracks and crevices occurred mostly in women with pour breastfeeding techniques, poor milk flow or duct obstruction [7].

Mastitis is defined by the WHO as an inflammatory condition of the breast which may be accompanied by infection or not. It is described to occur in about $6.6-33 \%$ women [19]. Although various risk factors such as milk stasis duct obstruction, and engorgement likely exist, a more common theory is that poor attachment of the infant to the breast leads to cracking of the nipple epithelium, which creates a retrograde path with inflammation and entry point for infection [19]. Most breast infections are due to Staphylococcus Aureus which is more prompt in causing local infection with abscess, than $\beta$-hemolytic Streptococcus which rather has more tendency to generalized infection [7]. These bacteria are found on the mother's skin surface, in the baby's throat and nostrils mainly, thus facilitating infection through breastfeeding [7]. Typical manifestations of mastitis are breast pains, redness, swelling and sometimes flu-like symptoms [20]. In this survey, infectious mastitis was generally easy to treat with Amoxicillin-Clavulanic acid association. Purulent breastmilk was a contraindication to breastfeeding, while continuous breastfeeding was recommended in the absence of purulent discharge in women under effective antibiotics therapy. This served for reducing milk stasis and inflammation [21, 22]. Nevertheless, severe infection with Methicillin-Resistant Staphylococcus Aureus may lead to death when poorly managed [7]. Therefore, breast abscess may complicate poorly managed acute bacterial mastitis in $4.8-11 \%$ cases, following delayed treatment or inefficient antibiotic therapy [7].

The lactating adenoma is a benign tumor, mainly caused by physiological changes occurring during lactation. It is similar to other physiological breast impairments such as fibroadenoma, tubular adenoma, or lobular hyperplasia [23, 24]. Lactating adenoma is a tumor made of epithelioid hyperplasia alone, contrary to other resembling tumors which may contain varying amounts of connective tissue proliferation. Lactation adenoma is softer in consistence and filled with breastmilk [7]. It often disappears at the end of lactation and relapses are quite rare [25].

\section{Conclusion}

There are considerable physiological changes occurring in the postpartum period which may favor breast pathologies. Such disorders are a source of worry for the mother and the medical staff, as a result of the impact they have on the breastfeeding process. Most of these disorders are secretion anomalies of which initial agalactorrhea is the most predominant. However, painful inflammatory and/or infectious pathologies represented by nipple crevices, breast engorgement, inverted nipples, mastitis and abscess, are frequent as well. All of these disorders are essentially benign in nature and favored by inadequate breastfeeding practices. The awareness of these conditions, their prevention by good hygiene and adequate breastfeeding techniques such as proper nipple and areolar positioning into the baby's mouth, and their effective management with painkillers and antibiotics when need be may help to protect the breastfeeding process, with favorable repercussions on infant development.

\section{Author Contributions}

Authors participated in all steps of the study.

\section{Funding}

Private.

\section{Conflict of Interest}

The authors declare that they have no competing interest.

\section{Acknowledgements}

To the Yaoundé Gynaeco-Obstetric and Paediatric Hospital authorities and all collaborators to this project. 


\section{References}

[1] Victora C, Sankar M, Rollins N, Murch S, Krasevec J, Horton $\mathrm{S}$, et al. Breastfeeding in the 21st century: epidemiology, mechanisms, and lifelong effect. Lancet. 2016; 387 (10017): 475-90.

[2] World Health Organization. Effect of breastfeeding on infant and child mortality due to infectious diseases in less developed countries: a pooled analysis. WHO collaborative study team on the role of breastfeeding on the prevention of infant mortality. Lancet. 2000; 355 (9202): 451-5.

[3] Patel A, Bucher S, Pusdekar Y, Esamai F, Krebs N, Goudar S, et al. Rates and determinants of early initiation of breastfeeding and exclusive breast feeding at 42 days postnatal in six low and middle-income countries: a prospective cohort study. Reprod Health. 2015; 12 (1): 2-9.

[4] Mullany L, Katz J, Li Y, Khatry S, LeClerq S, Darmstadt G. Breast-feeding patterns, time to initiation, and mortality risk among newborns in southern Nepal. J Nutr. 2008; 138 (3): 599-603.

[5] Baker E, Sanei L, Franklin N. Early initiation of and exclusive breastfeeding in large-scale community-based programmes in Bolivia and Madagascar. J Health Popul Nutr. 2006; 24 (4): $530-9$.

[6] Edmond K, Zandoh C, Quigley M, Amenga-Etego S, OwusuAgyei S,, Kirkwood B. Delayed breastfeeding initiation increases risk of neonatal mortality. Pediatrics. 2006; 117 (3): $380-6$.

[7] Ji Hoon Yu, Min Jeong Kim, Hyonil Cho, Hyun Ju Liu, SeiJun Han, Tae-Gyu Ahn. Breast diseases during pregnancy and lactation. Obstet Gynecol Sci 2013; 56 (3): 143-159.

[8] Bimerew A, Teshome M, Kassa G. Prevalence of timely breastfeeding initiation and associated factors in Dembecha district, North West Ethiopia: a cross-sectional study. Int Breastfeed J. 2016; 11: 28.

[9] Constance A, Gewa M, Monica O, Lauren S. Determinants of Early Child-Feeding Practices Among HIV-Infected and Non infected Mothers in Rural Kenya. J Hum Lact. 2011; 27 (3): 239-49.

[10] Odent M. Césariennes. Questions, effets, enjeux. Alerte face à la banalisation. Le Souffle d'Or. Barret-sur-Méouge: Elsevier Masson; 2005. 200 p.

[11] Stone K, Wheeler A. A review of anatomy, physiology, and benign pathology of the nipple. Ann Surg Oncol 2015; 22: $3236-3240.16$.

[12] Silva JR, Carvalho R, Maia C, et al. Rusty pipe syndrome, a cause of bloody nipple discharge: Case report. Breastfeed Med 2014; 9: 411-412.

[13] Kline TS, Lash SR. The bleeding nipple of pregnancy and postpartum period: A cytologic and histologic study. Acta Cytol 1964; 8: 336-340.

[14] Mitchell KB, Johnson HM, Eglash A. ABM Clinical Protocol \#30: Breast Masses, Breast Complaints, and Diagnostic Breast Imaging in the Lactating Woman. Breastfeed Med. 2019; 4 (4).

[15] Santos Kamilia Juliana da Silva, Santana Gessica Silva, Vieira Tatiana Oliveira, Santa Carlos, Antonio de Souza Teles, Gingliani Elsa Regina Justo, Vieira Graciete Olivieira. Prevalence and factors associated with cracked nipples in the first month of postpartum. BMC Pregnancy and Childbirth. 2016; 16 (1): 209.

[16] Williams Obstetrics (24 $4^{\text {th }}$ ed). Mc Grow-Hill Professional. 2014. pp. Chapter 37. ISBN 978-0-07-179893-8.

[17] Mangesi Lindeka, Zakarija-Grkovic, Irena. Treatments for breast engorgement during lactation. The Cochrane Database of Systematic Reviews. 2016. CD006946.

[18] Geffroy D, Doutriaux-Dumoulins I. Clinical abnormalities of the nipple-areola complex: Diagn Interv Imaging. 2015; 96: 1033-1044.

[19] Kvist LJ, Larsson BW, Hall-Lord ML, Steen A, Schalen C. The role of bacteria in lactational mastitis and some considerations of the use of antibiotic treatment. Int Breastfeed J. 2008; 3: 6.

[20] Joshi S, Dialani V, Marotti J, Mehta TS, Slanetz PJ. Breast disease in the pregnant and lactating patient: radiologicalpathological correlation. Insights Imaging (2013) 4: 527-538.

[21] Scott-Conner CE. Diagnosing and managing breast disease during pregnancy and lactation. Medscape Womens Health 1997; 2: 1 .

[22] Stafford I, Hernandez J, Laibl V, Sheffield J, Roberts S, Wendel G Jr. Community-acquired methicillin-resistant Staphylococcus aureus among patients with puerperal mastitis requiring hospitalization. Obstet Gynecol. 2008; 112: 533-7.

[23] Dehner LP, Hill DA, Deschryver K. Pathology of the breast in children, adolescents, and young adults. Semin Diagn Pathol 1999; 16: 235-47.

[24] Saglam A, Can B. Coexistence of lactating adenoma and invasive ductal adenocarcinoma of the breast in a pregnant woman. J Clin Pathol 2005; 58: 87-9.

[25] Hogge JP, De Paredes ES, Magnant CM, Lage J. Imaging and Management of Breast Masses During Pregnancy and Lactation. Breast J 1999; 5: 272-83. 